\title{
MANFRED STINGL \\ Construction of coupling-resurgent amplitudes in quantum field theory
}

\author{
Annales de la faculté des sciences de Toulouse $\sigma^{e}$ série, tome 13, \\ no 4 (2004), p. 659-682 \\ <http://www.numdam.org/item?id=AFST_2004_6_13_4_659_0>
}

(C) Université Paul Sabatier, 2004, tous droits réservés.

L'accès aux archives de la revue «Annales de la faculté des sciences de Toulouse » (http://picard.ups-tlse.fr/ annales/) implique l'accord avec les conditions générales d'utilisation (http://www.numdam.org/conditions). Toute utilisation commerciale ou impression systématique est constitutive d'une infraction pénale. Toute copie ou impression de ce fichier doit contenir la présente mention de copyright.

\section{NumDam}

Article numérisé dans le cadre du programme Numérisation de documents anciens mathématiques http://www.numdam.org/ 


\title{
Construction of coupling-resurgent amplitudes in quantum field theory
}

\author{
MANFRED STINGL ${ }^{(*)}$
}

\begin{abstract}
We describe a formal solution with resurgent couplingconstant dependence for the basic probability amplitudes of a strictly renormalizable and asymptotically free quantum field theory. We briefly review established facts about the form of the equations of motion and the small-coupling expansions for these amplitudes, emphasizing that operator-product expansions lead to resurgent-symbol formal representations of coupling dependence. We discuss why the resurgent symbols cannot be used directly in the equations of motion, and present an answer to this problem in the form of a quasi-perturbative expansion, based on resummation of the resurgent symbols in their nonperturbative direction through a sequence of rational approximants with respect to the couplingnonanalytic mass scale $\Lambda$. We sketch the distinctive mechanism, tied to the ultraviolet loop divergences in the equations of motion, by which the nonperturbatively modified zeroth quasi-perturbative orders (generalized Feynman rules) establish themselves self-consistently, and emphasize that it restricts formation of these zeroth orders rigorously to the finite set of primitively divergent vertex functions.
\end{abstract}

RÉSUMÉ. - Nous décrivons une solution formelle dont la dépendance en la constante de couplage est résurgente pour les amplitudes de probabilité de base d'une théorie quantique des champs strictement renormalisable et asymptotiquement libre. Nous passons brièvement en revue les faits établis sur la forme des équations du mouvement et les développements à faible couplage pour ces amplitudes, en accentuant le fait que les développements de produits d'opérateurs ("operator product expansions") mènent à des représentations formelles du type symbole résurgent pour la dépendance par rapport au couplage. Nous indiquons pourquoi les symboles résurgents ne peuvent être employés directement dans les équations du mouvement, et présentons une solution à ce problème sous la forme d'un développement quasi-perturbatif, basé sur la resommation des symboles résurgents dans leur direction nonperturbative à travers une suite d'approximations rationnelles par rapport à l'échelle de masse $\Lambda$ (elle-même non-analytique par rapport au

(*) Univ. Münster, Inst. für Theoret. Physik, D-48149 Münster (Westf.), Germany. E-mail: stingl@uni-münster.de 
couplage). Nous esquissons le mécanisme distinctif, lié aux divergences ultraviolettes, par lequel les termes quasi-perturbatifs d'ordre 0 modifiès non-perturbativement (règles de Feynman généralisés) s'établissent de façon cohérente, et soulignons que cela restreint la formation de ces termes d'ordre 0 rigoureusement à l'ensemble fini des sommets primitivement divergents.

\section{Generalities}

This talk deals with quantum field theory (QFT), and more specifically with the dependence of the probability amplitudes generated by QFT on the coupling strength. The problem I would like to address is really only at the outermost doorsteps of resurgence theory: the problem of how to construct a formal solution to the equations of motion for those amplitudes, providing a formal representation of their resurgent coupling dependence. I do think, however, that such a construction is a necessary first step before one can think of applying the more advanced concepts and techniques of resurgent analysis that we owe to Jean Écalle, and I hope to explain why already this purely formal first step is nontrivial due to the peculiarly complicated structure of the equations of motion.

At the end of this contribution, I have included only a few references immediately pertinent to this formal solution. The reason is that the last of these references [3] already offers a detailed four-page bibliography on the issues touched upon in this seminar, which it would be pointless to duplicate; so I am assuming your permission in directing you to this source for background information.

I will begin with a rapid review of some concepts, notations, and known or generally accepted facts about the basic probability amplitudes of QFT and their small-coupling expansions.

\section{Small-coupling expansions of QFT vertex functions}

In QFT, the amplitudes of interest are the so-called correlation functions: scalar products, taking values in the complex numbers, over the Hilbert space of the quantized-field system, and more precisely average or expectation values,

$$
\begin{aligned}
G_{N}\left(x_{1} \cdots x_{N}\right):= & \left(\Omega, \hat{\varphi}\left(x_{1}\right) \cdots \hat{\varphi}\left(x_{N}\right) \Omega\right), \\
& -660-
\end{aligned}
$$


of products of $N$ quantized-field operators $\hat{\varphi}$, at $N$ different four-dimensional spacetime points $x_{i}$, in the physical vacuum state $\Omega$. (In keeping with present-day usage in QFT, the $x_{i}$ will not be taken in Minkowski but in a four-dimensional Euclidean space, and amplitudes and their equations will be studied entirely in that Euclidean domain, with real-world Minkowskian amplitudes to be regained only in the end, provided certain general conditions are met, by analytic continuation in the fourth coordinate.) The set of all $G_{N}$ 's, with $N \in \mathbf{N}$, is the rough equivalent of the Schrödinger wave function in quantum mechanics - it represents the complete information about the system, and in particular all observable quantities of the QFT can be extracted from them, such as the rest masses and quantum numbers of particles, and the amplitudes for reactions between them.

In place of the $G_{N}$ we will mostly consider the equivalent set of proper vertex functions $\Gamma_{N}$, or their Fourier transforms $\tilde{\Gamma}_{N}$, which without going into their formal definition may be characterized as the simplest building blocks from which the full $G_{N}$ or $\tilde{G}_{N}$ may be pieced together purely algebraically - by multiplication, inversion, and addition. (In particular, the two-point vertex $\tilde{\Gamma}_{2}$ is just the inverse, up to a minus sign, of the two-point correlation $\tilde{G}_{2}$.) Each function

$$
\tilde{\Gamma}_{N}\left(k_{1} \cdots k_{N} ; g^{2}(\mu) ; \cdots ; \mu\right)_{R}
$$

depends on a set of $N$ four-dimensional wave-number or momentum arguments $k_{1} \cdots k_{N}$, obeying the restriction $k_{1}+\cdots+k_{N}=0$ due to translational invariance of the vacuum $\Omega$, so there are really only $N-1$ independent wave-number vectors. It also depends on a dimensionless renormalized coupling constant $g^{2}$, characterizing the strength of the interaction of the quantized fields with each other and with themselves, and whose physical values are real and positive: it is this dependence in which we will mainly be interested in the following. The dots in (2:2) stand for possible dependence on other variables, such as externally specified mass parameters, that we will ignore (in particular, we will consider massless theories). Finally there is dependence, both explicit and implicit through $g^{2}$, on a parameter $\mu$, as well as a label $R$, on which I will comment shortly.

To calculate these functions dynamically, one needs two types of input:

(i) An action functional - an essentially classical object, although it may include auxiliary terms necessary for quantization, and which in the QFT's of interest to particle physics has the rough structure

$$
S[\varphi]=S_{2}[\varphi]+g_{0} S_{3}[\varphi]+g_{0}^{2} S_{4}[\varphi] .
$$

The $S_{2}$ term is bilinear in the fields $\varphi$ and by itself would describe a set of noninteracting fields (a "free QFT"); it is in this term that externally 
specified mass terms, if any, would be present. The $S_{3}$ and $S_{4}$ pieces, being respectively tri- and quadrilinear, bring in the interactions among fields, and are therefore preceded by one or two powers of a bare coupling constant, $g_{0}$.

(ii) A renormalization scheme, denoted $R$ in (2.2), which is a set of prescriptions for dealing with the notorious problem of ultraviolet (UV) divergences that arise when writing and solving naively the quantum equations of motion for the action (2.3). For this talk, as in almost all analytic work on QFT these days, the dimensional schemes will be used, which regulate the UV divergences by calculating in an artificially reduced spacetime dimension,

$$
D=4-2 \varepsilon \quad(\varepsilon>0) .
$$

Divergences in correlation or vertex functions can then be isolated as terms with poles $\varepsilon^{-l}, l \in \mathbf{N}$, as the "dimensional regulator" $\varepsilon$ is taken to its physical value of zero, and can be compensated by including suitable counterterms in the action (2.3) so as to define finite parts of amplitudes. An important point about any such scheme is that it will always introduce, in the manner to be made precise by eq. (2.9) below, an arbitrary "renormalization mass scale" $\mu$, to which we already referred in (2.2); thus renormalization initially creates a whole family of QFT's differing by their $\mu$ values, and physical observables must evidently be sought among the class of "renormalization-group invariants", quantities independent of the arbitrary choice of $\mu$.

A second point is that in the dimensional continuation (2.4), the bare coupling $g_{0}$ acquires a mass dimension

$$
\left[g_{0}\right]=\frac{4-D}{2}=\varepsilon,
$$

whereas the renormalized coupling $g^{2}$ parametrizing the quantities $(2.2)$ is kept dimensionless by definition.

Given these two pieces of input, one may set up the quantum equations of motion for the $G_{N}$ or $\Gamma_{N}$, named after Dyson and Schwinger, which take the schematic form

$$
\tilde{\Gamma}_{N}=\underbrace{\tilde{\Gamma}_{N}^{(0) p e r t}}_{\text {from } S}+\{g_{0}^{2} \underbrace{\Phi_{N}\left[\tilde{\Gamma}_{2}, \cdots \tilde{\Gamma}_{N+1}, \tilde{\Gamma}_{N+2}\right]}_{\text {UV-divergent integrals }}\}_{R},
$$

for $N \in \mathbf{N}$. For each $\Gamma_{N}$ there is a nonlinear interaction functional, $\Phi_{N}$, preceded by at least two powers of the bare coupling $g_{0}$, and consisting of momentum-space integrals over products of $\tilde{\Gamma}$ 's up to $\tilde{\Gamma}_{N+1}$ (and, if an $S_{4}$ term is present in (2.3), to $\tilde{\Gamma}_{N+2}$ ). This coupling of each $\tilde{\Gamma}_{N}$ to the next 
higher ones is referred to as hierarchical, and one thus faces an infinite hierarchy of coupled nonlinear integral equations for the basic amplitudes $\tilde{\Gamma}_{N}$ - a problem rather different from the finite sets of differential or difference equations to which one is accustomed in classical dynamical systems, or even in one-particle quantum mechanics, and it is this peculiarly involved structure that renders the search for even purely formal solutions nontrivial. The integrals in $\Phi_{N}$ exhibit the UV divergences mentioned above at large wave numbers and thus require definition, as the notation in (2.6) indicates, through the renormalization scheme $R$. The first term in (2.6), the perturbative zeroth-order or bare vertex $\tilde{\Gamma}_{N}^{(0) \text { pert }}$, is nothing but the coefficient in the corresponding $S_{N}$ term of (2.3), and is therefore nonzero only for $N=2,3,4$. It is thus specified by the essentially classical action, and in this sense one may view the system (2.6) as describing the evolution of the quantum-field amplitudes away from their classical limits through quantum effects induced by the interaction.

Figure 1 shows, in the diagrammatic notation devised by physicists to avoid the writing of such unwieldy systems, the beginnings of a typical Dyson-Schwinger hierarchy - the three equations for $\tilde{\Gamma}_{N}$ 's with $N=2,3,4$, denoted by blobs with $N$ external legs (the $D^{-1}$ on the 1 . h. s. of the first equation is identical to $-\tilde{\Gamma}_{2}$ ). In each equation the r.h.s. starts with the corresponding $\tilde{\Gamma}_{N}^{(0) \text { pert }}$ term, followed by interaction terms whose closedloop topology signals the presence of a momentum integration, and which together constitute the quantum-effects functional $g_{0}^{2} \Phi_{N}$ of eq. (2.6). These "loop integrals" may be viewed as a direct expression of the superposition principle of quantum theory - each loop term specifies a class of intermediate states, labeled by some relative four-momentum, that can contribute to the quantum effects of a given $\tilde{\Gamma}_{N}$, and the integration expresses the fact that all these contributions have to be added up coherently. I will detail the translation of a typical interaction term of Fig. 1 into an analytical integral formula in sect. 3 below.

The simplest idea for generating a formal solution to the system (2.6) is, of course, to iterate it around the zeroth-order terms $\tilde{\Gamma}_{N}^{(0) \text { pert }}$. Since the interaction terms are preceded by a $g_{0}^{2}$ factor, it seems clear (we shall see the loophole in this conclusion below) that each iteration step generates another factor of $g_{0}^{2}$, which the renormalization process $R$ turns into $g^{2}$, and so one ends up with a formal power-series expansion in the renormalized coupling, the perturbation expansion:

$$
\tilde{\Gamma}_{N}^{(p e r t)}\left(\{k\} ; g^{2}(\mu) ; \mu\right)=\sum_{p=0}^{\infty} \tilde{\Gamma}_{N}^{(p) p e r t}(\{k\} ; \mu)\left(g^{2}(\mu)\right)^{p} .
$$


Manfred Stingl

(2 A)

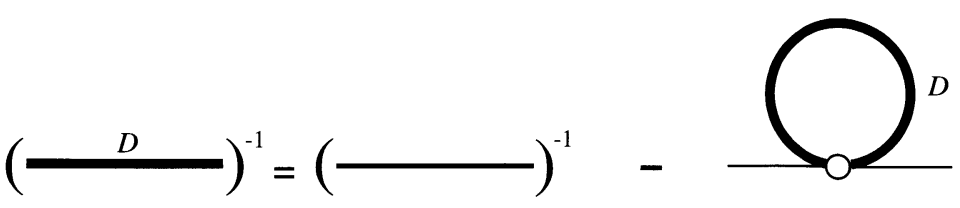

(2 C)

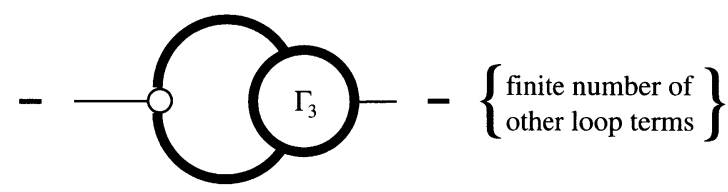

(3 A)

(3 B)
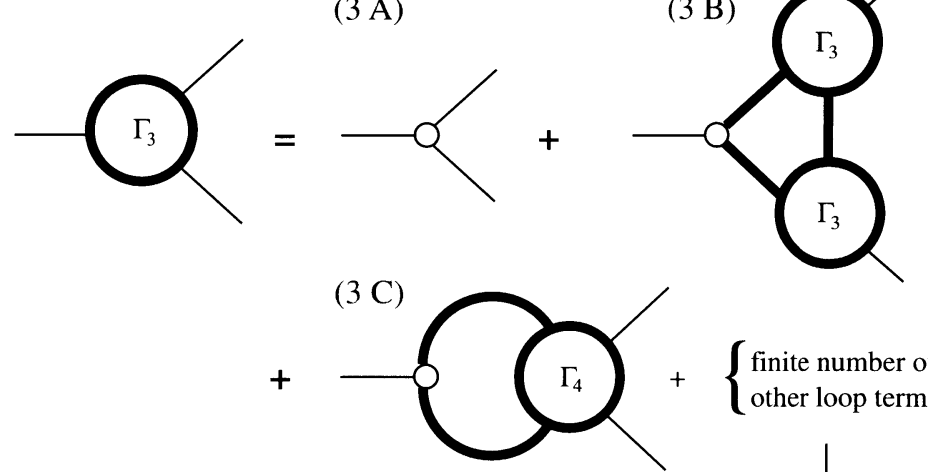

$\left\{\begin{array}{l}\text { finite number of } \\ \text { other loop terms }\end{array}\right\}$

(4 A)

(4 B)
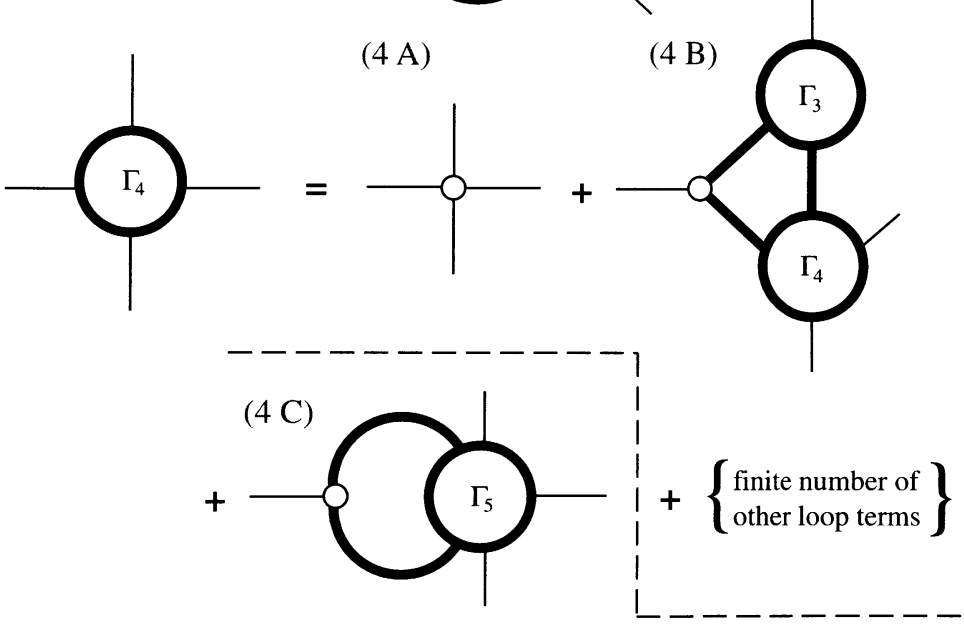

Figure 1. - First three equations of a typical Dyson-Schwinger hierarchy 
The individual contributions to the order- $p$ coefficient function $\tilde{\Gamma}_{N}^{(p) \text { pert }}$ can be enumerated conveniently by the method of Feynman graphs, and since (here I am simplifying a bit) the number of such graphs turns out to grow like $p$ ! at large $p$, the perturbation expansion is found to be a divergent, asymptotic series:

$$
\left|\tilde{\Gamma}_{N}^{(p) \text { pert }}\right| \propto\{\# \text { of order- } p \text { Feynman graphs }\} \propto p !
$$

In favorable cases, where the coupling $g^{2}(\mu)$ is sufficiently small at the scales $\mu$ dictated by the physics context, it may nevertheless exhibit loworder semiconvergence good enough for accurate calculations of quantum effects on observables. But even where this is not the case, the expansion (2.7) has dominated (and restricted) the analytic treatment of QFT to an extent that can hardly be overestimated.

The tendency to regard "the sum of the series" as synonymous with the exact solution of QFT was temporarily reinforced when it was proved in the 1970s that in (typical representatives of) the so-called superrenormalizable QFT's - model theories mostly living in spacetime dimensions $D \leqslant 3$ - the perturbation expansions, while divergent, are nevertheless summable, i.e. associated with unique, sectorially analytic functions of $g^{2}$. (Reconstruction of those functions has then been done by various, essentially classical methods, such as Padé approximants in $D=1$ or Borel-Laplace transform in $D=2$ and 3.) The superrenormalizable QFT's possess a special simplicity in that the $N=2$ functions $\tilde{G}_{2}$ (and, if Lorentz-scalar fields are present, the $N=1$ functions $\tilde{G}_{1}$ ) are the only "primitively divergent" ones: it is only these that develop their own characteristic UV divergences and call for specific counterterms, whereas all UV divergences occurring in higher $\tilde{\Gamma}$ 's can always be identified as contributions to such a $\tilde{G}_{2}$ appearing in a loop integrand; so renormalizing the $\tilde{G}_{2}$ basically renormalizes the whole system. (As an additional simplification, UV divergence turns out to be present in only a few low perturbation orders $p$, in contrast to realistic QFT's where it must be dealt with for all $p$ ). In such theories, then, the perturbation expansion, while divergent, still essentially encodes "the whole truth".

Unfortunately, the direction suggested by these results turned out to be misleading. In the realistic, four-dimensional QFT's relevant to present-day particle physics, which are "strictly" or "marginally" renormalizable rather than superrenormalizable, the perturbation expansion is known not to be summable; it does not permit unique reconstruction of amplitudes. Among the obstacles that have been identified, the most serious are the so-called infrared renormalons, classes of perturbation terms that produce, in the Borel transform with respect to $g^{2}$, an infinite sequence of poles on the real positive Borel axis. Now this fact alone, as I learned from Jean Écalle at 
this conference, would not nowadays be regarded by the resurgence theorist as precluding resummation; the technique of "uniformizing averages" does still allow one in such cases to construct a Laplace transform adapted to the natural context in which the series arises. But my point here is that for the QFT perturbation series such an effort is really unnecessary - the infrared renormalons are an entirely spurious phenomenon, artefacts of an illegitimate restriction of the small-coupling expansion, which simply disappear when the more general coupling dependence possible in the strictly renormalizable theories is taken into account consistently.

To identify this more general coupling dependence, one may start by asking what the fundamentally new ingredients are that distinguish the strictly renormalizable, realistic QFT's from the superrenormalizable ones and may be responsible for more involved coupling structure. Inspection shows that there is really only one such feature: not only the $\tilde{\Gamma}_{2}$ but also the $\tilde{\Gamma}_{3}$ and $\tilde{\Gamma}_{4}$ functions are now primitively divergent, and require their own renormalizations through counterterms. Since these evolve, through quantum effects, from the $g_{0} S_{3}$ and $g_{0}^{2} S_{4}$ terms of the action (2.3), it is plausible that this entails also a renormalization of the bare coupling $g_{0}$, a renormalization usually written in the form

$$
\underbrace{g_{0}^{2}(\varepsilon)}_{\text {mension } 2 \varepsilon}=\mu^{2 \varepsilon} \cdot \underbrace{g^{2}(\mu)}_{\begin{array}{c}
\text { dimensionless } \\
\text { and finite }
\end{array}} \cdot \underbrace{Z_{\alpha}\left(g^{2}(\mu), \varepsilon\right)}_{\begin{array}{c}
\text { dynamical } \\
\text { scale factor }
\end{array}} \cdot
$$

Since $g_{0}^{2}$ has mass dimension $2 \varepsilon$, we can connect it to the renormalized $g^{2}$, which is finite as $\varepsilon \rightarrow \infty$ and dimensionless by definition, only by splitting off a power $2 \varepsilon$ of some mass scale $\mu$, and since the theory so far has no distinguished scale, it is at this point that we get saddled with the arbitrary mass $\mu$ that we noted already in eq. (2.2). Finally, we need a dynamically determined scale factor $Z_{\alpha}$, the coupling-renormalization constant, whose $\varepsilon$ dependence is adjusted, order by order in a perturbation expansion in $g^{2}$, to cancel UV-divergent quantum effects on the coupling, and keep $g^{2}$ finite.

Now since observable quantities predicted by a QFT are not supposed to depend on the arbitrary choice of the mass $\mu$, we find ourselves forced to readjust the renormalized coupling $g^{2}$ every time we change $\mu$ so as to keep the observables constant: our $g^{2}$ has become a "running" coupling, dependent on $\mu$. Indeed the modern way of encoding the coupling renormalization (2.9) is by specifying the function describing this running, the 
renormalization-group ( $R G)$ beta function,

$$
\beta(g(\mu)):=\mu \frac{d}{d \mu} g(\mu)=-\varepsilon g-\underbrace{\beta_{0}}_{\text {scheme-independent }} g^{3}-\underbrace{\beta_{1}} g^{5}-\beta_{2} g^{7}-\cdots
$$

Its perturbative expansion is found to have, apart from a term $-\varepsilon g$ that goes away in the removal-of-regulator limit $\varepsilon \rightarrow 0$, a leading term $-\beta_{0} g^{3}$, whose coefficient $\beta_{0}$ depends only on the number and symmetry properties of the fields in the theory. Also, $\beta_{0}$ turns out - like the $\beta_{1}$ of the nextto-leading term, but unlike the $\beta_{n}$ with $n \geqslant 2$ - to be independent of the renormalization scheme $R$ adopted. Let me state without elaboration that it is the sign of this one number, $\beta_{0}$, that controls a deep structural property of the theory: the QFT is "asymptotically free", that is, its coupling $g^{2}(\mu)$ tends to zero slowly like an inverse logarithm at large scales $\mu$, exactly if $\beta_{0}>0$. We will from now on restrict attention to the strictly renormalizable and asymptotically free theories, since the non-abelian gauge theories that form the backbone of present-day particle physics belong to this class.

Strictly speaking, the commonly used notation $g^{2}(\mu)$ is nonsensical the dimensionless $g^{2}$ cannot possibly be a function of a single dimensionful variable. There must still be some other, fixed mass scale by which the $\mu$ can be divided in order to build a dimensionless quantity. Indeed it is straightforward to see that eq. (2.10) implies the existence of such a scale: write it as $d \mu / \mu=d g / \beta(g)$ and integrate over any interval $\left[\mu_{1}, \mu_{2}\right]$ of the $\mu$ scale. You find that the quantity

$$
\Lambda:=\mu \exp \left\{-\int_{g_{1}}^{g(\mu)} \frac{d g^{\prime}}{\beta\left(g^{\prime}\right)}\right\}
$$

is the same at any $\mu_{1}$ and $\mu_{2}$ and therefore $\mu$-independent: the so-called $R G$ invariant mass scale. (It still depends, through the lower integration limit $g_{1}$ as well as through the higher $\beta_{n}$ coefficients in $\beta\left(g^{\prime}\right)$, on the scheme $R$ adopted.) It is in this remarkable object - a scale nowhere visible in the classical action, but which the theory has created spontaneously through the regularization of its UV-divergent quantum effects - that the abovementioned compensation takes place between direct $\mu$ dependence and the indirect one through $g(\mu)$ : dimensionful observables of a classically massless QFT must be expressed in terms of $\Lambda$.

It is important to realize that the invariant scale (2.11) is not just a whimsical theoretical construct but has considerable observational significance: the QFT of the structure of the proton and neutron, Quantum Chro- 
modynamics (QCD), does have mass terms in its action (2.3) for the two types of fermion fields ("up quark" and "down quark" fields) that form the nucleons, but those masses are known to be orders of magnitude too small to explain the measured nucleon masses. The latter, and therefore almost all of the rest mass in the material world around us, are therefore to about 99 percent of the type of eq. (2.11).

In the physical spacetime dimension of $\varepsilon=0$, we now find by using $(2.10)$,

$$
\Lambda=\mu \exp \left\{-\frac{1}{2 \beta_{0} g^{2}}\left[1+O\left(g^{2}\right)\right]\right\},
$$

the prototype non-analytic coupling dependence that escapes representation by even a divergent power-series expansion around $g^{2}=0$. Let me stress that (2.12) is really nothing but a turned-around form of (2.10) and therefore a direct expression of coupling renormalization. Since the latter, as we emphasized above, is the only fundamentally new ingredient in the strictly renormalizable theories, it is plausible already at this point that the "strongly nonperturbative" quantity $(2.12)$ will be the only fundamentally new building block, in addition to the powers $\left(g^{2}\right)^{p}$ of the perturbative expansion (2.7), in the coupling dependence of realistic QFT amplitudes.

Although in what follows we will occasionally refer to the quantity (2.12) by the established epithet of "exponentially suppressed as $g^{2} \rightarrow 0+$ " because of its exponential factor, it is significant that this factor never appears alone, as do exponentially small factors in many other mathematical and physical contexts, but only in combination with dimensionful factors $\mu$ so as to form the invariant mass (2.12). The term "exponentially suppressed" therefore only applies in a purely formal and unphysical limit - the limit where we imagine $g^{2}$ tending to zero while keeping the $\mu$ factor constant. In physics, there is no way of achieving this, since the two quantities are correlated by construction so as to keep the product (2.12) invariant. The $\Lambda$ scale of Quantum Chromodynamics - not to speak of the one of electroweak theory which is a thousand times larger - has nothing exponentially small about it; it is a massive real-world presence.

How does $\Lambda$ appear in the correlation or vertex functions? An answer is contained in an asymptotic expansion introduced even before the invariant mass scale had been identified, the operator-product expansion (OPE). To explain this notion in the simplest case, consider the two-point correlation, eq. (2.1) at $N=2$. The central idea here is to expand the product $\hat{\varphi}\left(x_{1}\right) \hat{\varphi}\left(x_{2}\right)$ of field operators over the basis set

$$
\begin{aligned}
& \left\{\mathcal{O}_{i}^{(n)}(x) / i=1, \cdots, l(n)\right\}:=\text { set of all local operators at } x \\
& \text { (elementary or composite) of mass dimension } n \quad\left(\mathcal{O}^{(0)}=11\right),
\end{aligned}
$$


where $x=\frac{1}{2}\left(x_{1}+x_{2}\right)$, and to use translation invariance of the vacuum state $\Omega$. The result is an expansion,

$$
G_{2}\left(x_{1}, x_{2}\right)=\sum_{n=0}^{\infty} \sum_{i=1}^{l(n)} W_{i}^{(n)}\left(x_{1}-x_{2} ; g^{2} ; \mu\right) \cdot\left(\Omega, \mathcal{O}_{i}^{(n)}(0) \Omega\right),
$$

in terms of "vacuum condensates", the vacuum averages of the basis operators. Thus the basic hypothesis underlying the OPE is one of completeness of the set (2.13) of local operators in the space of field-operator products clustering around $x$. To my knowledge, there is at present no rigorous proof of this completeness statement; indeed since the "composite operators" (products of elementary fields and their spacetime derivatives at the same point $x$ ) are singular objects, requiring their own UV renormalizations beyond those of the primitively divergent correlations, much work may be needed to even clarify with respect to what topology or metric such a completeness statement may hold. At present, the belief of physicists in the OPE is therefore based more on the good success of certain semi-empirical applications of it. However, even apart from such applications, there is a degree of plausibility to it, since in a theory based on a finite number of fields as basic degrees-of-freedom, it is difficult to see what basis elements should be available for the expansion of products around a point other than the set of all local composites at that point.

The essential property of (2.14) is that the "Wilson coefficients", $W^{(n)}$, are objects perturbatively calculable as formal series in $g^{2}$. Indeed the $n=0$ term, containing the identity $\mathcal{O}_{1}^{(0)}=11$ and thus the vacuum norm $(\Omega, \Omega)=$ 1 , is nothing but the perturbation expansion of $G_{2}$ :

$$
W_{1}^{(0)}=G_{2}^{(p e r t)} .
$$

Thus the OPE is an explicit display of things that are lacking in the perturbation series and come in addition to it. Moreover, since the basis operators $\mathcal{O}_{i}^{(n)}$ and their vacuum condensates have increasing mass dimensions $n$, it is no surprise that the essential spacetime dependence of $W_{i}^{(n)}$ turns out to be of the form $\left[\left(x_{1}-x_{2}\right)^{2}\right]^{\frac{n}{2}-1}$, apart from logarithmic corrections coming with the higher perturbation terms. Actually, in the scalar correlations to which we restrict ourselves here for simplicity, only even- $n$ terms occur, so we may from now on write $2 n$ instead of $n$ and state that the corresponding coefficients $\tilde{V}_{i}^{(n)}$ for the Fourier-transformed two-point vertex $\tilde{\Gamma}_{2}$ behave as

$$
\tilde{V}_{i}^{(n)} \propto\left(k^{2}\right)^{1-n} .
$$

Now in a massless theory the only mass scale available for the vacuum condensates is the RG-invariant $\Lambda$ of $(2.11 / 12)$ - they cannot be powers of the 
arbitrary $\mu$, since this would make observable particle masses, determined by poles of $\tilde{G}_{2}$ or zeroes of $\tilde{\Gamma}_{2}$ in the $k^{2}$ plane, proportional to $\mu$. Therefore

$$
\left(\Omega, \mathcal{O}_{i}^{2 n}(0) \Omega\right) \propto \Lambda^{2 n} \propto\left(\mathrm{e}^{-\frac{1}{\beta_{0} g^{2}}}\right)^{n} .
$$

Combining these insights, we may write the OPE for the $\tilde{\Gamma}_{2}$ amplitudes as

$$
-\tilde{\Gamma}_{2}\left(k ; g^{2} ; \mu\right)=k^{2} \sum_{n=0}^{\infty}\left[\sum_{p=0}^{\infty} a_{n, p}\left(g^{2}\right)^{p}\right] \underbrace{\left(\frac{\mu^{2}}{k^{2}}\right)^{n} \mathrm{e}^{-\frac{n}{\beta_{0} g^{2}}}}_{\propto\left(\frac{\Lambda^{2}}{k^{2}}\right)^{n}} .
$$

The formal small- $g^{2}$ expansion for the strictly renormalizable theory therefore takes the form known as a resurgent symbol, a double expansion in terms of powers and of "exponentially small" factors. The symbol's support is on the equidistant points

$$
s_{n}=\frac{n}{\beta_{0}} \quad(n=0,1,2, \cdots),
$$

a property it shares with many of the resurgent symbols arising as formal integrals of ordinary differential equations.

(I have again been simplifying a bit: actually there are extra fractional powers, $\left(g^{2}\right)^{-\left(\frac{n \beta_{1}}{\beta_{0}^{2}}\right)}$ in (2.18) that arise from the $\beta_{1}$ term of $(2.10)$. This does not, of course, place the expansion (2.18) outside of resurgence theory; it merely says that the singularities of its Borel-major transform are not of the simplest type. Also, the $a_{n, p}$ coefficients in truth are not strictly constant with respect to $k^{2}$ but have a weak $k^{2}$ dependence through terms $\left[\ln \left(k^{2} / \mu^{2}\right)\right]^{q}$ with $q \leqslant p$, the type always associated with perturbative developments.)

A crucial property of expansion (2.18) follows directly from the fact, emphasized above, that the "exponentially small" factors never appear in isolation but always in the form of powers of the invariant-mass combination (2.11), and that as a result the coefficients must follow the pattern (2.16): the resurgent symbols in $g^{2}$ automatically become large-momentum expansions in powers of $\Lambda^{2} / k^{2}$. This tight linkage between coupling and momentum dependences has nontrivial consequences. Not the least of these is that "physical" singularities of $\tilde{G}_{N}$ 's with respect to $k^{2}$ arguments - branch lines at timelike $k^{2}$ associated with the possibility of scattering and reaction processes - translate into complex- $g^{2}$-plane branch lines, the so-called 
t'Hooft singularities, whose form does not allow for a finite sectorial opening angle, as $g^{2} \rightarrow 0+$, in the region of $g^{2}$ analyticity. Again I think such phenomena are not alien to resurgence theory, but they do mean that here one is dealing from the outset with one of the more complicated situations for which some of the theorems and techniques usually based on sectorialanalyticity hypotheses may need to be adapted.

An interesting side-aspect of representation (2.18) is that it leads to a reformulation of the question of completeness for the OPE. Although resurgent symbols like (2.18) already represent a much larger class of functions than, say, the Borel-summable power series of superrenormalizable theories, we cannot at present (to my knowledge) exclude the possibility that they still miss certain terms in the vertex functions. Such terms then would have to be what resurgence theory calls rapidly decreasing functions, objects decreasing faster than any exponential as $g^{2} \rightarrow 0+$. (One of the few things that can be said is that the aforementioned t'Hooft singularities in the $g^{2}$ plane do not necessarily entail such non-uniqueness; a counterexample is offered in ref. [3].) This would mean that there are missing terms in the OPE, i. e. that the expansion of operator products in the basis (2.13) is incomplete. If on the other hand we accept completeness of the OPE as plausible, we are led to expect that the symbol (2.18), and analogous small$g^{2}$ expansions for the other vertex functions, will be summable into unique resurgent functions of the coupling. As far as I can see, this linkage, whose underlying mechanism is still completely in the dark and all the more intriguing, is not yet widely appreciated. The idea seems to be widespread that the $\Lambda$ of (2.12) is just one among many possible coupling dependences. By contrast we emphasize here that it is quite likely, and certainly compatible with known facts, that the resurgent symbols of type (2.18) account for the totality of the strongly nonperturbative coupling dependence.

\section{Presummation of resurgent symbols}

The OPE represents a deep structural statement, but it is not a replacement for a dynamical theory. The latter must, in some way or other, proceed from exact equations of motion like (2.6). It is then natural to ask, why not go ahead and insert the resurgent-symbol formal series into those equations to determine their coefficients, as one would do when solving differential equations? It is again a peculiarity of QFT that this standard procedure turns out to be impossible.

To understand why, take a look at a typical loop-integral term in one of the interaction functionals $\Phi_{N}$ of eqs. (2.6), namely, the term marked (2 C) in Fig. 1 on the r. h. s. of the $N=2$ equation. Its analytic expression 
is developed in Fig. 2 below: the small and large circles translate into bare and full three-point vertices respectively - each carrying one factor of $g_{0}$, which makes for the overall $g_{0}^{2}$ in (2.6) -, while the two heavy lines translate into two-point functions $\tilde{G}_{2}$ ("propagators") at four-momenta $q$ and $k-q$ adding up to the conserved total momentum $k$ flowing through the equation. The closed-loop topology, finally, translates into the instruction to integrate over the "loop momentum" $q$ of the intermediate configuration.

It is essential that the integration should run over all of $q$ space, and therefore also over the points $q=0$ and $q=k$ where the momenta in the $\tilde{G}_{2}$ and $\tilde{\Gamma}_{3}$ functions of the integrand vanish. We saw that as a result of the tight linkage between the $\Lambda^{2}$ and momentum dependences, the resurgent symbols of type (2.18) for these functions are unavoidably large-momentum expansions - here, expansions in powers of $\Lambda^{2} / q^{2}$ and $\Lambda^{2} /(k-q)^{2}$. But such expansions obviously cannot be used in integrals extending down to $q^{2}=0$ and $(k-q)^{2}=0$, where their errors become arbitrarily large and indeed lead to arbitrarily strong infrared divergences. If one were to use them blindly, in the vague hope that our dimensional regularization (2.4) may regulate these errors too, one would meet disappointment: the $\Lambda^{2}$ terms in (2.18) would produce contributions to the loop proportional to the parameter integrals

$$
\int_{0}^{1} \frac{d x}{x^{n-2+\varepsilon}(1-x)^{m-2+\varepsilon}} \quad(m, n \in \mathbf{N}) \text {, }
$$

and we would need arbitrarily large negative $\varepsilon$ 's to regulate all of these, while UV regularization requires us to keep $\varepsilon>0$. There is, of course, nothing surprising about this; one simply has been using an approximation in a context where its validity breaks down. It is clear that simple perturbation theory would never run into this problem - since it knows no $\Lambda$ scale, it does not know of the $n \geqslant 1$ terms in (2.18), but only of powers of $g^{2}(\mu)$ which for the momentum integration are mere constant parameters.

In what follows I describe an answer to this problem that is probably not unique. Also, in its present form it is heuristic and formal, and not yet a rigorous mathematical construction. I do think, however, that it is workable, and that in principle it provides a coherent and systematic framework in which to address certain questions inherently beyond the domain of applicability of the perturbation expansion. On the mathematical side, I hope it can lead to interesting conjectures and perhaps be of some help in identifying worthwhile research problems. 


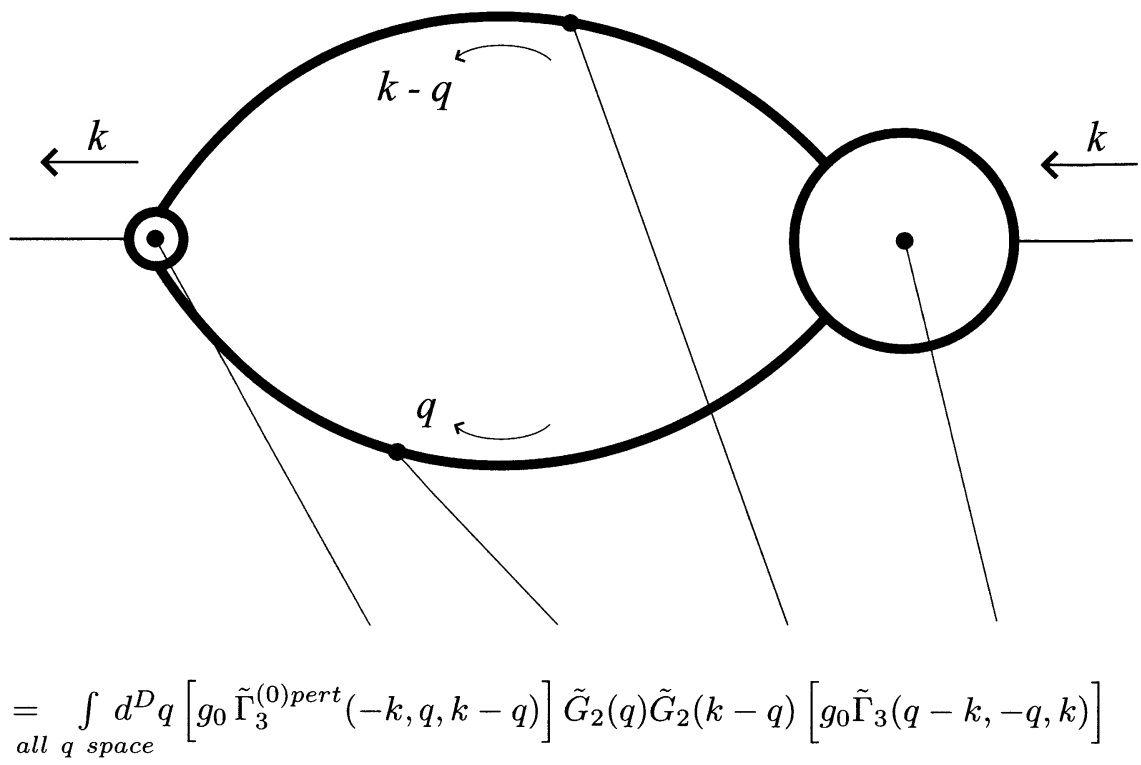

Figure 2. - Term (2C) from first equation of Fig. 1 and its analytic expression

The starting point is to recognize that in order to have a formal representation of $\tilde{\Gamma}_{N}$ 's that can be determined dynamically from the integral equations, one obviously needs at least a partial continuation-throughresummation of the resurgent symbol, namely a resummation of its "nonperturbative direction", the $n$ summation proceeding in powers of $\left(\Lambda^{2} / k^{2}\right)^{n}$ at fixed $p$. This will automatically provide a continuation of $k^{2}$ dependence down to the region $k^{2} \approx 0$, so that the representation can sensibly be used in the loop integrals of the dynamical equations. The net result will be a quasi-perturbative expansion,

$$
\tilde{\Gamma}_{N}=\sum_{p=0}^{\infty} \tilde{\Gamma}_{N}^{(p)}(\Lambda)\left(g^{2}\right)^{p}
$$

which looks like a perturbation series, except that the coefficients (exemplified here for the case (2.18) of a two-point vertex),

$$
\tilde{\Gamma}_{N}^{(p)}=\text { a resummation of } \sum_{n=0}^{\infty} c_{n, p}\left(\frac{\Lambda^{2}}{k^{2}}\right)^{n}, \quad\left(c_{n, p} \propto a_{n, p}\right)
$$


contain the nonanalytic coupling dependence (2.12) that cannot be accounted for by the power series. It is not necessary to devise such resummations for all $p$ : all one needs to do to get started is to have one for the $p=0$ terms, the zeroth quasi-perturbative orders

$$
\begin{aligned}
\tilde{\Gamma}_{N}^{(0)} & =\text { resummation of }\left\{c_{0,0}+\sum_{n=1}^{\infty} c_{n, 0}\left(\frac{\Lambda^{2}}{k^{2}}\right)^{n}\right\} \\
& =\tilde{\Gamma}_{N}^{(0) \text { pert }}+\tilde{\Gamma}_{N}^{(0) \text { nonp }} .
\end{aligned}
$$

They are easier to deal with because this series is likely to possess a finite radius of convergence: by (2.14), the growth of the coefficients $c_{n, 0}$ is mainly controlled by the growth in the number $l(n)$ of local composite operators of mass dimension $n$, and that number - in contrast to the number of Feynman diagrams contributing to the perturbative coefficients $c_{0, p}$ - grows "only" exponentially:

$$
\begin{aligned}
& \left|c_{n, 0}\right| \propto\{\# l(n) \text { of local operators of } \\
& \text { mass dimension } 2 n\} \leqslant(\text { const. })^{n}
\end{aligned}
$$

Once these $n$-resummed zeroth orders $\tilde{\Gamma}_{N}^{(0)}$ have been determined or at least systematically approximated, we may entrust to the dynamical equations themselves the job of generating the $p \geqslant 1$ quasi-perturbative corrections $\tilde{\Gamma}_{N}^{(p)}$ by iteration around these nonperturbatively improved starting solutions, rather than around the $\widetilde{\Gamma}^{(0) p e r t}$ 's of perturbation theory.

From the point of view of resurgence theory, there are two extra motivations for this quasi-perturbative strategy that deserve mention:

(i) By keeping the $n=0$ (perturbative) and $n \geqslant 1$ (nonperturbative) parts together in one function at each $p$, one avoids the production of spurious Borel-plane singularities that arise separately in both parts and cancel in their sum. The "infrared renormalons" mentioned earlier are of this type; their presence in the perturbative Borel-minor transform is entirely due to the illegitimate omission of the nonperturbative terms.

(ii) Model studies indicate that the quasi-perturbative expansion (3.2), viewed as a series in $g^{2}$ with $\Lambda$ treated formally as an unrelated parameter, is Borel summable in the conventional sense (without averaging techniques). I have allowed myself to present such a model-amplitude study, which also illustrates point (i), in sect. 2.4 of ref. [3]; this model may also be of interest because it allows the quasi-perturbative resummation to be carried out completely in terms of a class of functions - the polylogarithms - that have attracted interest also in other mathematical contexts. 
The practical construction of the $\tilde{\Gamma}_{N}^{(0)}$ functions, in the scheme described here, is done by the classical method of rational approximants with respect to $\Lambda^{2}$ - simply because rational approximants have proven themselves in other physics contexts to be a robust tool for continuing germs of analytic functions beyond a finite convergence radius. The asymptotic-freedom property of the theory fixes the relative degrees of their numerator and denominator polynomials in advance; thus for the 2-point vertex functions we have the approximating sequence

$$
-\Gamma_{2}^{[r, 0)}(k)=\frac{\tilde{\zeta}_{0}\left(k^{2}\right)^{r+1} \cdots+\tilde{\zeta}_{r+1}\left(\Lambda^{2}\right)^{r+1}}{\left(k^{2}\right)^{r}+\cdots+\tilde{\eta}_{r}\left(\Lambda^{2}\right)^{r}}=\frac{N_{2}^{[r]}\left(k^{2}, \Lambda^{2}\right)}{\Delta_{2}^{[r]}\left(k^{2}, \Delta^{2}\right)}
$$

characterized by their denominator degrees $r=0,1,2, \cdots$ These, as well as their inverses $\tilde{G}_{2}(k)$, are automatically also rational with respect to the momentum variable $k^{2}$, which allows them to be handled in the loop terms of the integral equations. For the higher $\tilde{\Gamma}_{N}$ 's depending on more than one scalar momentum argument, the analogous property is not automatic; for these one therefore relies on an additional analyticity assumption which allows e.g. the three-point function $\tilde{\Gamma}_{3}^{(0)}$ (three scalar variables from two independent momentum vectors) to be written as a triple Cauchy integral

$$
\int_{C} d s_{1} \int_{C} d s_{2} \int_{C} d s_{3} \frac{\rho\left(s_{1}, s_{2}, s_{3}\right)}{\left(k_{1}^{2}+s_{1} \Lambda^{2}\right)\left(k_{2}^{2}+s_{2} \Lambda^{2}\right)\left(k_{3}^{2}+s_{3} \Lambda^{2}\right)},
$$

plus analogous terms depending on only two or one of the variables. (Here $C$ is a denumerable set of real or complex-conjugate paths in the $s$ planes.) Discretization of such a representation leads to approximants of the form

$$
\tilde{\Gamma}_{3}^{[r, 0)}\left(k_{1}^{2}, k_{2}^{2}, k_{3}^{2}\right)=\frac{N_{3}^{[r]}\left(k_{1}^{2}, k_{2}^{2}, k_{3}^{2} ; \Lambda^{2}\right)}{\Delta_{3}^{[r]}\left(k_{1}^{2}, \Lambda^{2}\right) \Delta_{3}^{[r]}\left(k_{2}^{2}, \Lambda^{2}\right) \Delta_{3}^{[r]}\left(k_{3}^{2}, \Lambda^{2}\right)}
$$

with a numerator polynomial $N_{3}$ and a factorizing denominator polynomial, which mirrors the factorizing structure of the Cauchy denominator. We do not write the corresponding approximants for $\tilde{\Gamma}_{4}$, with six scalar momentum arguments besides the $\Lambda^{2}$ variable, but we anticipate the remarkable fact, discussed in the next section, that one does not need to specify any zerothorder approximants for $N \geqslant 5$ functions.

There are two noteworthy features to these $\tilde{\Gamma}_{N}^{[r, 0)}$ approximants:

(i) Calculability: this property may be marginal from a conceptual point of view but is nevertheless very important for the working physicist. The loop integrals arising upon iteration of the coupled integral-equations system around the $\tilde{\Gamma}_{N}^{[r, 0)}$ can be evaluated by the existing and well-developed 
techniques of loop computation, since their integrands, while more complex than those of perturbation theory, are still rational in momenta.

(ii) Self-consistency: at any given level $r$, the set $\left\{\tilde{\Gamma}_{N}^{[r, 0)}\right\}$ are capable of self-reproduction in the coupled integral equations (2.6), up to $p \geqslant 1$ quasi-perturbative corrections $\tilde{\Gamma}_{N}^{[r, p)}$ that are simultaneously generated in the process, provided a finite set of algebraic self-consistency conditions are imposed on the coefficients of the approximants. It is this property that is decisive in establishing the quasi-perturbative series (3.2) as a formal solution to the equations of motion. The property is not trivial, since at first sight the $g_{0}^{2}$ factor in front of the interaction functionals $\Phi_{N}$ of (2.6) seems to ensure that only terms with at least $\left(g^{2}\right)^{1}$, but not with $\left(g^{2}\right)^{0}$, can be generated.

\section{Self-consistency of the generalized Feynman-rules}

I must naturally be brief here about the technical details of this selfconsistency result, and will therefore concentrate on the one essential mathematical element that makes it possible. Since during iteration we are now feeding into the $\Phi_{N}$ the $\tilde{\Gamma}_{N}^{(r, 0)}$ approximants with their ubiquitous $\Lambda$-scale terms, rather than the old $\tilde{\Gamma}^{(0) p e r t}$ 's, their loop integrals become capable of producing terms in which ultraviolet divergence, $\frac{1}{\varepsilon}$, and the noninteger power $\left(\Lambda^{2}\right)^{-\varepsilon}$ appear as factors. In these terms, then, the exact RG identity

$$
g_{0}^{2} \frac{1}{\varepsilon}\left(\Lambda^{2}\right)^{-\varepsilon}=\frac{1}{\beta_{0}}[1+O(\varepsilon, \varepsilon \ln \varepsilon)]
$$

applies, whose r.h.s. is rigorously independent of $g^{2}(\mu)$ and therefore of $\mu$, and finite as $\varepsilon \rightarrow 0$ with the scheme-independent value of $1 / \beta_{0}$. This identity follows [1] by combining the exact representation (2.11) of $\Lambda$ with an exact integral representation of $g_{0}^{2}$ derived (from (2.9), (2.10), and the UV-finiteness condition for $\left.g^{2}(\mu)\right)$ in 1973 by t'Hooft:

$$
g_{0}^{2}=\mu^{2 \varepsilon} g^{2}(\mu) \exp \left\{-\int_{0}^{g^{2}(\mu)} \frac{d\left(g^{\prime 2}\right)}{g^{\prime 2}+\varepsilon \chi\left(g^{\prime 2}\right)}\right\}
$$

where

$$
\chi\left(g^{2}\right):=\frac{1}{\beta_{0}+\beta_{1} g^{2}+\cdots}=\frac{g^{3}}{-\beta(g, \varepsilon=0)} .
$$

While a combination of known RG results, the identity (4.1) has nontrivial consequences in our context because 
(i) one factor of $g^{2}$ gets "eaten": inspection shows that this is due to the behavior $\left(\Lambda^{2}\right)^{-\varepsilon} \propto 1 / g^{2}$ (at all $\varepsilon$ ) inherent in (2.11). Therefore terms of zeroth order in $g^{2}$ can be established despite the overall prefactor of $g_{0}^{2}$. One might say the "mistake" of perturbation theory is to overlook precisely this possibility: since perturbation theory misses the $\Lambda$ scale, it can never produce the combination (4.1);

(ii) one factor of $\frac{1}{\varepsilon}$ gets "eaten": inspection shows that this comes from the nonperturbative vanishing as $\varepsilon \rightarrow 0$ of the $Z_{\alpha}$ factor of (2.9), a property inherent in (4.2) and also known to t'Hooft thirty years ago. This exact property seems to have been sitting in the QFT archives as an oddity, since perturbation theory, which treats the $Z_{\alpha}$ by expansion in $g^{2}$ with UVdivergent coefficients, never gets confronted with it. In the nonperturbative environment discussed here, it appears naturally and acts in an interesting way: the compensation of UV divergence for the zeroth-order terms now proceeds through (4.1), rather than by the artifice of counterterms. Thus renormalization, while still basically perturbative - it depends upon the perturbative calculability of the $\beta$ function, and upon the property of our approximants not to produce worse-than-perturbative UV-divergence - is found to "work along a different route".

To briefly demonstrate the workings of (4.1) in the simplest case, consider again the two-point $(N=2)$ equation. On the one-loop level (first iteration), we replace the arguments in $\Phi_{2}$ by their zeroth-quasi-perturbativeorder approximants $\Gamma^{[r, 0)}$. Concentrate on the $(2 \mathrm{C})$ term of Fig. 1 as explained in Fig. 2. The input to the loop consists, first, of expression (3.6) which we now write in the partial-fraction form

$$
-\tilde{\Gamma}_{2}^{[r, 0)}=\zeta_{0} k^{2}+\zeta_{1} \Lambda^{2}+\sum_{s=1}^{r} \frac{\zeta_{s+1} \Lambda^{4}}{k^{2}+\eta_{s+1} \Lambda^{2}}
$$

(More precisely, we need its inverse, $\tilde{G}_{2}^{[r, 0)}$ ). Second, due to the hierarchical coupling, we need to put in the zeroth-order three-point vertex, approximated by (3.8), whose coefficient sets we denote by

$$
\left\{\xi^{i}\right\} \text { for } N_{3}^{[r]}, \quad\left\{\vartheta_{j}\right\} \text { for } \Delta_{3}^{[r]} \text {. }
$$

Upon feeding these into the loop and evaluating it by standard techniques, one finds that since a $\Delta_{3}^{[r]}\left(k^{2}\right)$ denominator from (3.8) comes outside the integral, the term can be written in a form resembling (4.4), namely 


$$
\begin{array}{r}
{\left[1+g_{0}^{2} R_{0}\left(\frac{k^{2}}{\Lambda^{2}}, \varepsilon\right)\right] k^{2}+\left[g_{0}^{2} R_{1}\left(\frac{k^{2}}{\Lambda^{2}}, \varepsilon\right)\right] \Lambda^{2}} \\
+\sum_{s=1}^{r} \frac{\left[g_{0}^{2} R_{s+1}\left(\frac{k^{2}}{\Lambda^{2}}, \varepsilon\right)\right] \Lambda^{4}}{k^{2}+\vartheta_{s+1} \Lambda^{2}}
\end{array}
$$

where the $R_{i}$ functions contain the $\{\zeta, \eta\}$ and $\{\xi\}$ sets of parameters polynomially, are UV-divergent (contain poles $\frac{1}{\varepsilon}$ ), and are otherwise "weakly" (i. e. logarithmically) $k^{2}$-dependent near $\varepsilon=0$. It is clear that this form becomes possible because of the "Cauchy structure" of (3.8), with its factorizing denominator.

For comparison with the input (4.4), we need to isolate the residues in (4.6) by the "subtraction"

$$
\begin{aligned}
R_{i}\left(\frac{k^{2}}{\Lambda^{2}}, \varepsilon\right) & =R_{i}\left(-\vartheta_{i}, \varepsilon\right) \\
& +\left[R_{i}\left(\frac{k^{2}}{\Lambda^{2}}, \varepsilon\right)-R_{i}\left(-\vartheta_{i}, \varepsilon\right)\right] .
\end{aligned}
$$

It turns out (and is familiar to the practitioner of QFT perturbation theory) that this also isolates the UV divergences, which are now contained in the first r. h.s. term of (4.7): in a renormalizable QFT, UV divergence is always located in a few low-order Taylor terms of the loop integrals with respect to external momenta. The result is

$$
\begin{aligned}
g_{0}^{2} R_{i}\left(\frac{k^{2}}{\Lambda^{2}}, \varepsilon\right)=g_{0}^{2} \frac{1}{\varepsilon}\left(\Lambda^{2}\right)^{-\varepsilon}\left[X_{i}(\{\zeta, \eta\},\{\xi\})+O(\varepsilon)\right] \\
+g_{0}^{2} \underbrace{\text { TTaylor remainder] }}_{\text {UV finite }}
\end{aligned}
$$

(The first two of the "subtraction points", $\vartheta_{0}$ and $\vartheta_{1}$, remain arbitrary but will not influence the determination of zeroth orders). In the first term, use of the exact identity (4.1) shows that there is really no coupling dependence left. If term (2C) of Fig. 1 were the only contribution to the $\Phi_{2}$ functional (this situation would actually occur for a fermionic two-point vertex), it would therefore be possible to reproduce the postulated zeroth order (4.4) by imposing the algebraic self-consistency conditions,

$$
\begin{gathered}
\eta_{s+1}=\vartheta_{s+1} \quad(s=1, \cdots, r) \\
\zeta_{s}=\delta_{s 0}+\frac{1}{\beta_{0}} X_{s}(\{\zeta, \eta\},\{\xi\}) \quad(s=0, \cdots, r+1) \\
-678-
\end{gathered}
$$


If there are more terms in $\Phi_{2}$, the conditions still take this form, but with the meaning of $\{\xi\}$ now expanded to include the coefficient sets of the other 2 - and 3-point vertices appearing in those terms.

Condition (4.9), in the notation of (3.6) and (3.8), enforces $\Delta_{2}^{[r]}=\Delta_{3}^{[r]}$, and this type of condition - a direct consequence of the hierarchical coupling - keeps reappearing in the next higher vertex equations of Fig. 1: for a given type of field $\hat{\varphi}$ in the correlation functions, the same denominator polynomial $\Delta^{[r]}$, at the momenta $k_{i}^{2}$ of all external legs of that type, is present in all zeroth-order vertices at level $r$. Looking back at (3.7), we see that beyond the context of rational approximation, this means that the same set $C$ of contours is involved in the momentum analyticity of all zeroth-order functions - a strong structural statement.

On the other hand the Taylor remainders in (4.8) are UV finite, so in these the $\varepsilon \rightarrow 0$ limit can be performed straight away. The special combination (4.1) now does not form; the prefactor of $g_{0}^{2}$ remains in isolation, and we must decide about how to treat its dependence on our basic resurgence variable, $g^{2}$. But since we have opted for a quasi-perturbative expansion (3.2), our only consistent choice is to use (2.9) as expanded in powers of $g^{2}$. On the one-loop level at which we are working, this amounts to using

$$
g_{0}^{2}=g^{2}\left[1+O\left(g^{2}\right)\right] \quad(\varepsilon=0) .
$$

This somewhat clumsy dichotomy in the treatment of $g_{0}^{2}$ is connected to the different treatments we have applied to the " $n$ direction" at $p=0$ (allorders resummation) and the " $p$ direction" (plain Taylor expansion) of the resurgent coupling dependence. The net result may be written

$$
\Gamma_{2}=\Gamma_{2}^{[r, 0)}+g^{2} \Gamma_{2}^{[r, 1)}+O\left(g^{4}\right),
$$

where $O\left(g^{4}\right)$ stands for terms from higher loop orders; thus we have the beginnings of expansion (3.2) established.

The mechanism seen here at work in the self-consistent generation of $\Lambda$-rational terms may now be applied to the next higher $(N \geqslant 3)$ vertex equations. It is obvious from (4.1) that this mechanism is not only tied to the presence of loop integrals (and therefore a pure quantum effect) but also to their renormalizable UV divergences (and therefore a pure quantum-field effect). This has some noteworthy consequences:

First, in higher iterations it turns out that the iteration or loop order $l$ and the quasi-perturbative order $p$, which in pure perturbation theory coincide, are now no more identical: an $l$-loop term of $g_{0}^{2} \Phi_{N}$ is found to contribute to all quasi-perturbative orders $\Gamma^{[r, p)}$ with $p \leqslant l$. In particular, 
the self-consistency equations of type (4.10) receive corrections involving higher powers of $1 / \beta_{0}$,

$$
\zeta_{s}=\delta_{s 0}+\frac{1}{\beta_{0}} X_{s}^{(l=1)}+\frac{1}{\beta_{0}^{2}} X_{s}^{(l=2)} \ldots,
$$

arising through eq. (4.1) from the combination of a $\left(g_{0}^{2}\right)^{l}$, present in front of any $l$-loop contribution, with the "maximally divergent" portions, containing $\left(\frac{1}{\varepsilon} \Lambda^{-2 \varepsilon}\right)^{l}$, of the $l$-fold momentum integrals. Thus the self-consistency conditions determining the parameters of the rational approximants get themselves refined in successive loop orders. While expansion (4.13) does not create new problems of principle - the maximally divergent parts are known from RG arguments to form, at large $l$, only a geometric subseries with finite convergence radius of the total, factorially divergent $l$ expansion - , the fact that at present we can calculate only in low orders $l$ is definitely the most unsatisfactory theoretical aspect of and practical restriction on the method at this time.

Second, and perhaps most remarkably, it is now straightforward to see that the formation of zeroth quasi-perturbative orders $\Gamma_{N}^{[r, 0)}$ is rigorously restricted to the few primitively divergent functions with $N=2,3,4$. This follows from the fact that in the $N \geqslant 5$ functions which are not primitively divergent, the number of $g_{0}^{2}$ prefactors in front of a loop contribution is always larger (by at least one, the difference increasing with $N$ ) than the number of $\frac{1}{\varepsilon} \Lambda^{-2 \varepsilon}$ factors produced, so that the mechanism of eq. (4.1) can produce only $p \geqslant 1$ quasi-perturbative corrections, but no $p=0$ contributions. This finiteness result, which now truly justifies referring to the $\Gamma_{N}^{[r, 0)}$ as generalized Feynman rules, is one of the few exact statements that can be made about the method at present. It is all the more remarkable as it does not invoke any of the so-called decoupling approximations that are usually found necessary to truncate the infinite hierarchy (2.6) to a finite and tractable problem; it only relies on the privileged role conferred upon the primitively divergent functions by a mechanism operating from UV-divergent loops. (The decoupling approximations, to be sure, may still be useful strategies for dealing with the resummation of the $p$ series, which this talk will stop short of considering). 
Our considerations may be summed up in the statement:

At any level $r$ of rational approximation and any loop order $l$, the quasi-perturbative expansion, with zeroth orders $\Gamma_{N}^{[r, 0)}$ for $N \leqslant 4$ determined by self-consistency conditions of type (4.9) and (4.13), and with $p=1 \ldots l$ corrections generated by $l$-fold iteration, provides a formal solution to the coupled equations of motion up to corrections of order $l+1$, with resurgent coupling dependence arising from the dependence of coefficients $\Gamma^{[r, p)}$ on the nonperturbative mass scale $\Lambda$.

\section{Closing Remarks}

I will skip completely a discussion of the existing application to QCD [2], which is restricted to the $r=1$ and $l=1$ levels, deals only with the self-consistency problem of zeroth orders, and was intended mainly as a demonstration of technical feasibility. I would however like to briefly mention two types of problems which are inherently out of reach for ordinary perturbation theory but which the partially resummed, nonperturbative zeroth orders allow one to address in a coherent framework. The first is the calculation of qualitative changes in the zeroth-order particle spectrum, governed by the $\tilde{G}_{2}$ functions of the theory, such as shifts from perturbatively massless to massive excitations. The second is the generation of structures in the $\Gamma_{3}$ and $\Gamma_{4}$ functions whose symmetries differ from those in the $\Gamma^{(0) p e r t}$ (and therefore in the essentially classical action). These two effects, known respectively as dynamical mass generation and dynamical symmetry breaking, can both be produced in principle from the coupled equations of motion, and in an analytically transparent way, through the self-consistent formation of $\Lambda$-rational terms discussed above.

On the mathematical side, several of the problems arising in the context of QFT coupling dependence will by now be evident. As for "foundational" issues, quite apart from the specific formal solution described here, it would be urgent to know more about the questions (interrelated, as we have seen) of the completeness of the OPE and the summability of the corresponding resurgent symbols. In particular, it would be highly desirable to have sufficient summability conditions, analogous to the Nevanlinna-Sokal criterion for functions with ordinary Borel-summable expansions, that allow for the less-than-sectorial analyticity domains produced by the t'Hooft singularities. In the context of the formal solution discussed here, it would be important to have the conjecture of ordinary Borel summability for the 
quasi-perturbative expansion (3.2) confirmed or elucidated, to procure better justification of the rational approximation for the $\Gamma_{N}^{(0)}$ s, and to have the essentially geometric character of the series of type (4.13) established more rigorously. These zeroth-order self-consistency equations, by the way, are in themselves an interesting area of study - they represent a kind of simplified algebraic image of the complicated integral-equations system with its nonlinearities and hierarchical couplings, and the fact, emphasized above, that they form a strictly finite set may make them a less intimidating, more well-delineated object of investigation. A full description of the resurgent structure of QFT amplitudes with respect to the coupling is still a distant goal. On all these problems, physicists are likely to be in need of help - as you know, and as David Hilbert knew already a hundred years ago, physics is really much too difficult for the physicists. That observation, then, provides also my final apology for treating you to this heuristic mélange.

Acknowledgements. - I am grateful to Frédéric Fauvet and David Sauzin for extending to me an invitation to contribute to the Résurgence-2002 conference, and to Jean Ecalle for helpful remarks on Borel summability and for his friendly interest in the subject. I am indebted to Daniel Ebbeler for expert te $\chi$ ing and graphics.

\section{Bibliography}

[1] Stingl (M.). - A systematic extended iterative solution for quantum chromodynamics, Zeitschr. f. Physik A353, p. 423-445 (1996); E-print archive: hep-th/9502157 (1995).

[2] Driesen (L.), Kuhrs (J.) and Stingl (M.). - Extended iterative scheme for QCD: three-point vertices, Eur. Physical J. A4, p. 381-400 (1999); L. Driesen and M. Stingl, Extended iterative scheme for QCD: the four-gluton vertex, Eur. Physical J. A4; p. 401-419 (1999); E-print archive: hep-th/9808152 and hep-th/9808155 (1998).

[3] Stingl (M.). - Field-Theory Amplitudes as Resurgent Functions, hep-ph/0207349 